\title{
Incorporating Statistical Measures of Anatomical Variability in Atlas-to-Subject Registration for Conformal Brain Radiotherapy
}

\author{
Olivier Commowick ${ }^{1,2}$, Radu Stefanescu ${ }^{1}$, Pierre Fillard ${ }^{1}$, Vincent Arsigny ${ }^{1}$, \\ Nicholas Ayache ${ }^{1}$, Xavier Pennec ${ }^{1}$, and Grégoire Malandain ${ }^{1}$ \\ 1 INRIA Sophia - Epidaure Project, 2004 Route des Lucioles BP 9306902 Sophia \\ Antipolis Cedex, France \\ Olivier.Commowick@sophia.inria.fr \\ 2 DOSISoft S.A., 45-47 Avenue Carnot, 94230 Cachan, France
}

\begin{abstract}
Deforming a digital atlas towards a patient image allows the simultaneous segmentation of several structures. Such an intersubject registration is difficult as the deformations to recover are highly inhomogeneous. A priori information about the local amount of deformation to expect is precious, since it allows to optimally balance the quality of the matching versus the regularity of the deformation. However, intersubject variability makes it hard to heuristically estimate the degree of deformation. Indeed, the sizes and shapes of various structures differ greatly and their relative positions vary in a rather complex manner. In this article, we perform a statistical study of the deformations yielded by the registration of an image database with an anatomical atlas, and we propose methods to re-inject this information into the registration. We show that this provides more accurate segmentations of brain structures.
\end{abstract}

\section{Introduction}

Brain radiotherapy must achieve two goals: the complete irradiation of the tumor, and the preservation of certain critical structures (brainstem, eyes, optical tracts, etc.). By customizing the shape of the irradiation beam and modulating the irradiation intensity, conformal radiotherapy allows to optimize the irradiation of the tumor and the critical structures. The planning of conformal radiotherapy requires accurate localizations of the tumor and the critical structures. In existing planning systems, the segmentation of brain structures is manual and each structure has to be delineated in each slice of a 3D image (e.g. MRI). An automatic segmentation algorithm of all the critical structures in a patient image is then an invaluable tool for radiotherapy, and its main requirement is a precise delineation of the structures of interest.

In order to segment all these structures in a specific patient's image, we use an anatomical atlas (described in [1]) containing labels of the structures of the brain. The atlas was manually labeled from an artificial MR image (obtained from the BrainWeld ${ }^{1}$ ). The first step of the general segmentation method is a rigid

\footnotetext{
${ }^{1}$ See web site: http://www.bic.mni.mcgill.ca/brainweb/
} 
matching between the atlas and the patient MRI (usually $\mathrm{T} 1$ ). The recovered transformation is refined using non-rigid registration, and then applied to the atlas labelization in order to obtain a segmentation of the patient image.

Due to its multi-subject nature, the non-rigid registration problem is generally difficult. The topology of the brain, the shape of the ventricles, the number and shape of the sulci vary strongly from one individual to another. Thus, algorithms have to deal with the ambiguity of the structures to match and also to take into account the large variability of the differences between the two brains. The ideal transformation is smooth in some places, and has fine details in others. Registration algorithms based on a uniform regularization lead therefore to local misregistrations as they do not take into account this variability. In the literature, this problem has been explored in the following two ways.

On the one side, some registration algorithms using inhomogeneous regularization [23] were recently introduced. They apply a strong regularization where the local deformability is low, and a weak regularization where it is high. However, they use heuristic maps of the deformability. These approaches lead to better results than with uniform regularization, but heuristic models are generally too simple compared to the complexity of the deformability. As a result, the model is not accurate everywhere and there are still local misregistrations.

On the other side, some studies have been conducted on brain asymmetry [4] and on the variability of the cortex surface using a non-rigid registration algorithm 5]. Some studies have also used extrapolation of the variability information on sulci all over the brain [6]. However, to our knowledge, none of them has been used yet to guide in some way a non-rigid registration algorithm.

In this article, we introduce a framework to compute deformability statistics over a database of patient MRI. These statistics are in turn used to guide the regularization of the deformation field. In Section 2, we first introduce an algorithm which is able to take into account scalar or tensor information to guide the regularization. In Section 3. we present the pipeline used to compute the statistics and the two deformability measures we propose. Finally, we present experiments on our image database that demonstrate quantitatively better segmentations with the proposed method, and qualitatively much more consistent from an anatomical point of view.

\section{Incorporating Statistics}

The registration algorithm proposed in [7] recovers the transformation as an invertible dense displacement field. Given a target image $I$ and a source image $J$, the algorithm recovers the deformation field $U$ which makes the deformed source image $J \circ U$ be as similar as possible to the target image. The method consists in the optimization of a three-term criterion:

$$
E=\operatorname{Sim}(I, J \circ U)+\int_{x \in \Omega}\left\|k(x) \nabla \frac{\partial U}{\partial t}(x)\right\|^{2} d x+\int_{x \in \Omega}\|D(x) \nabla U(x)\|^{2} d x .
$$

The first term optimizes the similarity between the target and source image. The second term performs a regularization of the temporal derivative of the 
displacement field, similar to a fluid regularization of the velocity field in fluid mechanics. By locally weighting this "fluid" regularization with a scalar field $k$, 8] showed that the algorithm can be rendered robust with respect to potential pathologies in the subject image.

The third term of the criterion performs a regularization of the displacement field, thereby simulating an elastic-like behavior. This "elastic" regularization is weighted by a space-varying field $D$. If $D(x)$ is scalar, the regularization can model position-dependent deformations: the larger $D(x)$ is, the larger the local deformation can be. If $D$ is a tensor field, the deformability is position-dependent and direction-dependent: the amount of deformation allowed can be separately tuned along spatial directions. Indeed, when one performs a diagonalization of $D(x)$, its eigenvalues model the deformability along the directions represented by the eigenvectors of the tensors (see [7]).

This elastic-like regularization model can very well incorporate deformability statistics: if the deformations are known to be large in some place, $D(x)$ is assigned a low value. Conversely, a high $D(x)$ is assigned if the deformations are known to be locally low. Furthermore, if we also have directional information about the local deformations, $D$ can be a tensor. Its eigenvalues are large if the deformations along the corresponding eigenvectors are small, and vice versa.

\section{Computation of Statistical Variability Measures}

Our goal here is to build statistical measures of the deformability of the brain, that can be re-introduced as regularization maps $D(x)$ in the algorithm described above. To build the deformability statistics, we need a reference image on which we bring all the images. As choosing one image among the others in the database would introduce a bias in our approach, we use the simulated MRI of the atlas as our reference image. Then, all the images are brought to the atlas geometry using the algorithm described in Section 2 with uniform regularization, meaning that we do not make any assumption about the local deformability of each structure. This pipeline is illustrated on Fig. 1. Once the deformation fields are computed, we need a way to evaluate the mean deformability on the reference image: we now turn to the definition of scalar and tensor measures we propose.

\subsection{Scalar Statistics}

A good estimator of the local deformation caused by the mapping of the patient's geometry onto the atlas geometry is the Jacobian matrix $J$ of the deformation. Indeed, its determinant $|J(x)|$ indicates whether the region around voxel $x$ locally shrunk $(|J(x)|<1)$ or locally expanded $(|J(x)|>1)$.

Using only the determinant of the Jacobian matrix, it is clear that the contractions and expansions have not a symmetric influence when we compute statistics of the deformability: expansions will have a greater importance than contractions. We therefore propose the following expression based on the determinant:

$$
\operatorname{De} f_{j}(x)=\operatorname{abs}\left(\log \left(\left|J_{j}(x)\right|\right)\right)
$$




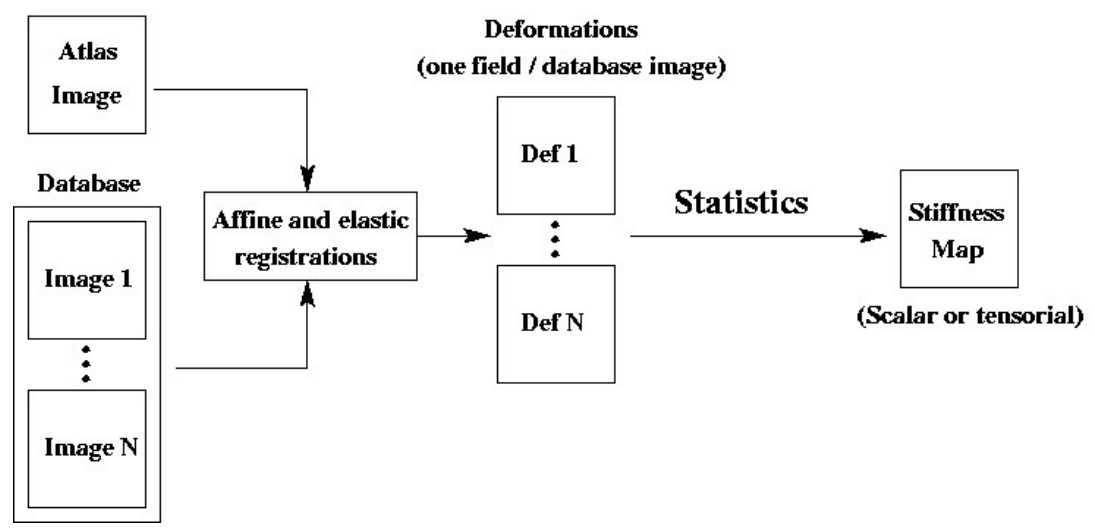

Fig. 1. Schematic view of the pipeline used to compute the deformability statistics. This scheme shows which major steps are done for computing our scalar or tensor stiffness map. (see text)

In this formula, the index $j$ corresponds to the patient while $x$ is the current voxel position. Taking the logarithm symmetrizes the influence of the deformations, thus avoiding to penalize the contractions. The absolute value insures that we have no compensation between contractions and expansions at voxels where both occur in different images. Finally, this distribution can be summarized by its mean value:

$$
\overline{\operatorname{Def}}(x)=\mathbb{E}\left(\operatorname{abs}\left(\log \left(\left|J_{j}(x)\right|\right)\right)\right) .
$$

This equation can also be seen as a dispersion measure associated to the median, i.e. a robust version of the standard deviation. However, as we said in Section 2 , the quantity of regularization in the algorithm is defined by a stiffness map $D(x)$, where $D(x) \in[0 ; 1]$ is a scalar map. The greater $D$ is, the stronger the regularization is and therefore the less the deformability is. We can see that $D$ is roughly equivalent to the inverse of the measure we defined above. To keep the value of $D$ bounded, we use the following formulation for the stiffness map with $\lambda>0: D(x)=1 /(1+\lambda \overline{D e f}(x))$.

\subsection{Tensor Statistics}

At this point, we have defined a scalar estimator of the mean deformability of the brain. However, the drawback of a scalar field is that we do not use all the information given by the deformation: we have not taken into account the directional information of the displacements. As the algorithm presented above allows us to perform anisotropic regularization using tensor fields, we would like to have an extension of the preceding formulation to compute tensor statistics of the brain deformability.

The tensor we propose here is based on the Jacobian matrix: $W_{j}(x)=$ $J_{j}(x) . J_{j}(x)^{T}$. This expression can be seen as a measure of the local deformation. A related idea was suggested in [9], but directly on the Jacobian matrix. 
However, using the symmetric deformation tensor $W$ allows us to compute simple and efficient (rigid-invariant) statistics.

As for the scalar field, we want to symmetrize the influence of the contractions and the dilatations. We therefore take the matrix logarithm of this expression and the absolute value of the resulting value. Let $W=R . \operatorname{Diag}\left(S_{i}\right) \cdot R^{T}$ be a diagonalization of a symmetric matrix (where $S_{i}$ are the eigenvalues of the matrix). Then, the matrix absolute value is defined by $\operatorname{abs}(W)=R \cdot \operatorname{Diag}\left(\operatorname{abs}\left(S_{i}\right)\right) \cdot R^{T}$. Finally, we define the measure of deformability by: $\Sigma_{j}(x)=\operatorname{abs}\left(\log \left(W_{j}(x)\right)\right)$. And the expression for the mean deformability is simply:

$$
\bar{\Sigma}(x)=\mathbb{E}\left(\operatorname{abs}\left(\log \left(W_{j}(x)\right)\right)\right) .
$$

Similarly to the scalar map, we use the following expression to obtain a bounded stiffness tensor map: $D(x)=(I d+\lambda \bar{\Sigma}(x))^{-1}$.

\section{Experiments and Results}

We used in our experiments a database of 36 patients with brain tumors at different grades and positions. For each patient three MRI have been acquired (T1, injected $\mathrm{T} 1$ and $\mathrm{T} 2$ ). All these patient images are registered on the atlas MRI following the process in Fig. 1, first by an affine global registration, then using a uniform elastic registration.

Once the deformation fields are obtained, we compute a mask of the region of interest for each image. This allows us to compute our statistical measures only on tissues that are present in both the patient and the atlas images. The problem is similar for tumor and surgical resections. The correspondences are not relevant in these regions as these tissues are not homologous in both images. Then, as we have $\mathrm{T} 1$ and $\mathrm{T} 2$ images of the patient, we segment the tumor and the surgical resection as in [8] and remove these segmentations from the mask. We are then able to compute deformability statistics on relevant regions using Eqs. (3) and (44). An example of the resulting stiffness maps and fractional anisotropy (FA is an index of the anisotropy of the tensor) for the tensor-based model are given in Fig. 2. As we compute our statistics only on imaged regions of each brain of the database, we do not have any information on some exterior regions of the atlas image, leading to some black regions in the FA map.

As we can see on Fig. 2, the statistical stiffness maps we computed are smoother than the heuristic model used in [7. We can notice that the heuristic assumptions made in [7] on the deformability of the ventricles are mostly verified. However, in some regions (like the ventricles boundaries), the tensor deformability map is highly anisotropic (see the FA map in Fig. 2). The statistical maps are also very different from the heuristic one in the region in front of the brainstem. This region is attributed a more elastic behavior than in the heuristic map, where it is set to almost 0, resulting in a fluid regularization. However, we can see in Fig. 3 that this assumption can result in relatively bad segmentations 

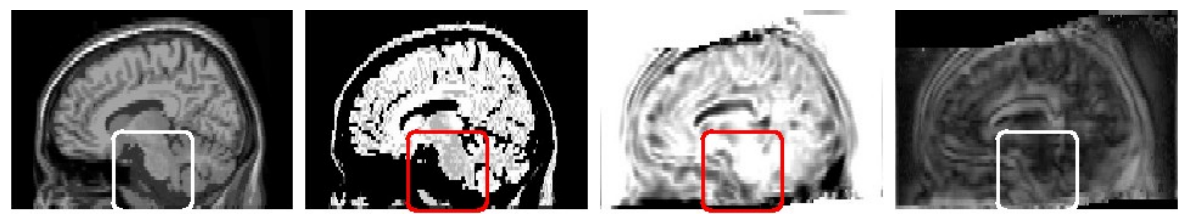

Fig. 2. A slice of the statistical maps obtained in our comparison. The frames show the brainstem and the area in front of it. From left to right: the simulated MRI of the atlas, the heuristic scalar map, the scalar map obtained using Eq. (3), the fractional anisotropy (FA) map obtained using Eq. (4) (see text).
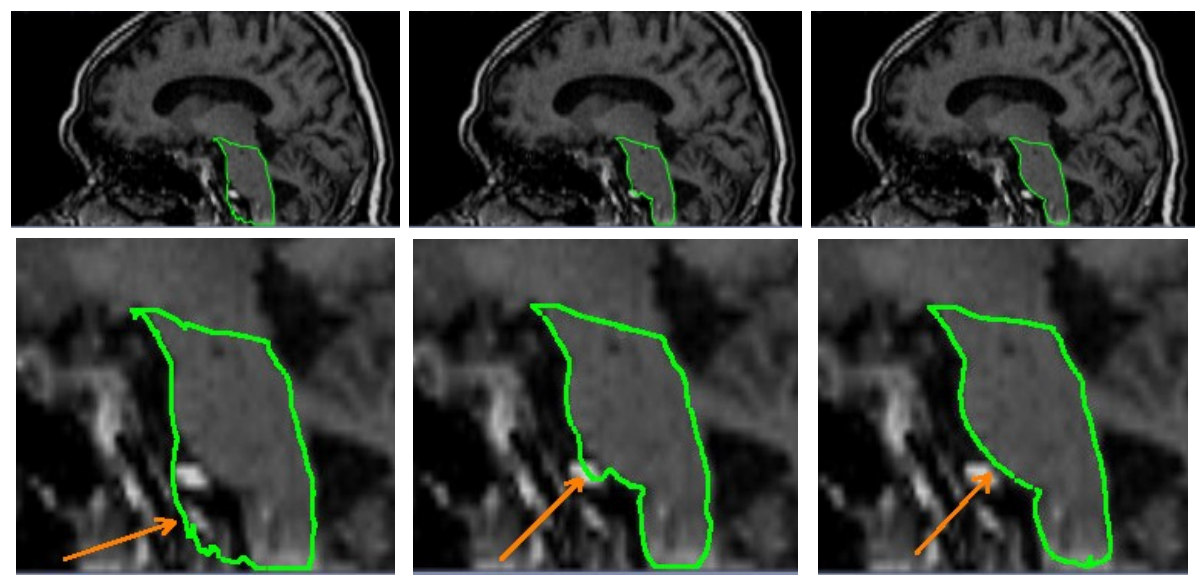

Fig. 3. Comparative results of the atlas-based segmentation. Top: Sagittal slice of the 3D patient image with the segmentation superimposed. Bottom: close-up on the brainstem area. From left to right: registration with the heuristic scalar map, with the statistical scalar map and with the statistical tensor map.

of the brainstem. In this case, the segmented structure often includes the artery just in front of it. This is due to the absence of matter in the atlas image leading to bad correspondences.

Using a fluid registration in this area, as proposed by the heuristic model, leads to non-anatomical deformations. In our scalar stiffness map as well as in its tensor equivalent, the region just in front of the brainstem is assigned to a higher value. Thus, we focus on an example of the automatic segmentation of the brainstem using the image of a patient that was not used to compute the statistics. We can see that the results are improved in this region as shown in Fig. 3. The segmentation is qualitatively more consistent. We can also see that taking into account a directional component in the regularization (tensor map) further improves the results as the artery in front of the brainstem is not anymore included in the segmentation (see arrows in Fig. 31). Finally, the registration process takes about one hour to run in sequential execution on an AMD Opteron $2 \mathrm{GHz}$, independently of the statistical model used. 
Table 1. Statistics obtained using the STAPLE algorithm [10]. Seven expert segmentations for each patient (Sens. stands for Sensitivity, Spec. for Specificity and dist. for the distance to the point (Sens. $=1$, Spec. $=1)$ ) (

\begin{tabular}{|l|c|c|c|c|c|c|c|c|c|}
\hline & \multicolumn{3}{|c|}{ Patient 1 } & \multicolumn{3}{c|}{ Patient 2 } & \multicolumn{3}{c|}{ Patient 3 } \\
\hline & Sens. & Spec. & Dist. & Sens. & Spec. & Dist. & Sens. & Spec. & Dist. \\
\hline Heuristic & 0.86 & 0.87 & 0.19 & 0.81 & 0.74 & 0.32 & 0.83 & 0.88 & 0.21 \\
\hline Scalar & 0.84 & 0.92 & 0.18 & 0.82 & 0.83 & 0.25 & 0.81 & 0.94 & 0.20 \\
\hline Tensor & 0.86 & 0.91 & $\mathbf{0 . 1 7}$ & 0.83 & 0.84 & $\mathbf{0 . 2 3}$ & 0.82 & 0.94 & $\mathbf{0 . 1 8}$ \\
\hline
\end{tabular}

At this point, we have seen that our method gives qualitatively better results than the method using a scalar heuristic stiffness map on one example. We also studied in more details the results on three other patient images for which seven experts have manually segmented the brainstem. For each patient image, we compute the ground truth segmentation (see [10]) from the expert segmentations. Then, we use this ground truth to compute the sensitivity and the specificity of each automatic segmentation. We also compute the distance to the best result achievable (Sensitivity $=1$, Specificity $=1$ ). The results are reported in Table1 and confirm our qualitative estimation: the results obtained by the scalar statistics map are slightly better than the ones obtained by the heuristic map. We verify also quantitatively that using an anisotropic regularization map gives better results than using only isotropic information.

\section{Conclusion}

In this article, we have described a method to incorporate statistics of the deformability of the brain into a non-rigid registration algorithm. We have introduced scalar (isotropic) and tensor-based (anisotropic) models to this end. For each method, we have detailed a symmetric measure of the deformations, on which we can perform consistent statistics. These statistics are also robust since we explicitly exclude the tumor and surgical resection parts of the patients images from the computation of the statistics.

The two ways of re-introducing statistics in the registration result in qualitative and quantitative improvement of the segmentations, the tensor-based model achieving the best results. However, concerning quantitative validation, only three patients were used with only one structure segmented manually by seven experts. We intend to extend this validation to more patients and to more structures in a near future.

The framework we have used has the advantage to be independent of the non-rigid algorithm used to compute the statistics. However, it is sensitive to potential systematic errors in the algorithm used to register images on the atlas image. To compute less biased statistics, it will be interesting to use other non-rigid algorithms (for example parametric algorithms as [11]) or to bring into correspondence manual delineations of structures. Ideally, in order to be as unbiased as possible, we would like to be able to fuse the information provided by several non-rigid registration algorithms into one single statistical map. 
Finally, this algorithm can be used on other regions of the body. This approach could bring a way to evaluate the local deformability on regions where it is difficult to have a good idea of the elasticity of the deformation.

\section{Acknowledgments}

This work was partially founded by ECIP project MAESTRO and ANRT. The authors are grateful to Dr. P.-Y. Bondiau for providing the MRI database and the brain atlas used for our experiments.

\section{References}

1. PY Bondiau, G Malandain, O Commowick, PY Marcy, S Chanalet, and N Ayache. Atlas-based automatic segmentation of MR images: Validation study on the brainstem in radiotherapy context. In $R S N A$, Chicago, 2004.

2. Hava Lester, Simon R. Arridge, Kalvis M. Jansons, Louis Lemieux, Joseph V. Hajnal, and Anjela Oatridge. Non-linear registration with the variable viscosity fluid algorithm. In Proc of IPMI;99, pages 238-251, 1999.

3. R. Stefanescu, X. Pennec, and N. Ayache. Grid powered nonlinear image registration with locally adaptive regularization. Med. Im. Analysis, 8(3):325-342, 2004.

4. A.W. Toga and P.M. Thompson. Mapping brain asymmetry. Nature Reviews Neuroscience, 4(1):37-48, January 2003.

5. P.M. Thompson, M.S. Mega, K.L. Narr, E.R. Sowell, R.E. Blanton, and A.W. Toga. Brain image analysis and atlas construction. In M. Fitzpatrick and M. Sonka, editors, Handbook of Medical Image Proc. and Analysis, chapter 17. SPIE, 2000.

6. P. Fillard, V. Arsigny, X. Pennec, P. Thompson, and N. Ayache. Extrapolation of sparse tensor fields: Application to the modeling of brain variability. In IPMI'05, LNCS. Springer, July 2005. To appear.

7. R. Stefanescu. Parallel nonlinear registration of medical images with a priori information on anatomy and pathology. PhD thesis, Université de Nice Sophia-Antipolis, March 2005.

8. R. Stefanescu, O. Commowick, G. Malandain, P.-Y. Bondiau, N. Ayache, and $\mathrm{X}$. Pennec. Non-rigid atlas to subject registration with pathologies for conformal brain radiotherapy. In Proc. of MICCAI'04, volume 3216 of LNCS, pages 704-711. Springer, 2004.

9. R.P. Woods. Characterizing volume and surface deformations in an atlas framework: theory, applications, and implementation. NeuroImage, 18(3):769-788, 2003.

10. Simon K Warfield, Kelly H Zou, and William M Wells. Simultaneous truth and performance level estimation (staple): an algorithm for the validation of image segmentation. IEEE Trans Med Imaging, 23(7):903-21, July 2004.

11. G.K. Rohde, A. Aldroubi, and B.M. Dawant. The adaptive bases algorithm for intensity based nonrigid image registration. IEEE TMI, 22:1470-1479, 2003. 